\title{
Long-Term IOP Lowering With Bimatoprost in Open-Angle Glaucoma Patients Poorly Responsive to Latanoprost
}

\author{
Sriram Sonty, ${ }^{1,2}$ Vikrant Donthamsetti, ${ }^{2}$ Gautam Vangipuram, ${ }^{2}$ and Afzal Ahmad ${ }^{2}$
}

\begin{abstract}
Purpose: The aim of this study was to study long-term intraocular pressure (IOP) lowering following a switch to bimatoprost in patients with open-angle glaucoma (OAG) not at target IOP while on latanoprost either as monotherapy or as polytherapy with other topical adjunctive agents.

Methods: A retrospective review of OAG patients, with $<20 \%$ IOP lowering from pretreatment baseline while on latanoprost either as monotherapy or in combination with adjunctive agents who were switched to bimatoprost, was conducted. Main outcome measures were mean IOP at 6, 12, and 24 months following the switch to bimatoprost and percent IOP lowering from baseline IOP before the switch.

Results: Records of 30 patients (30 right eyes [OD], 29 left eyes [OS]) were reviewed. Mean IOP preswitch was $23.1 \pm 4.3 \mathrm{mmHg}$ in OD and $22.3 \pm 3.8 \mathrm{mmHg}$ in OS. Postswitch to bimatoprost, IOP was significantly reduced $(P<0.005)$ at each of the time points studied and patients experienced additional IOP lowering ranging from 17.8 to $22.0 \%$ in OD and $15.0-24.0 \%$ in OS. Bimatoprost was well tolerated in all but 1 patient.

Conclusions: Significant additional long-term IOP lowering may be achieved by switching to bimatoprost in patients with open-angle glaucoma who are not at target IOP with latanoprost.
\end{abstract}

\section{Introduction}

I T IS ESTIMATED THAT BY THE YEAR 2020, there will be approximately 80 million people worldwide with glaucoma, and $74 \%$ of these patients will have open-angle glaucoma (OAG). ${ }^{1}$ Although glaucoma is a neurodegenerative disease, the only risk factor that can be modulated to slow its progression at present is intraocular pressure (IOP). Currently, the only available therapeutic option to treat glaucoma is pharmacologic or surgical IOP lowering.

Prostaglandin analogs are commonly used as first-line IOP-lowering agents in the treatment of OAG. Bimatoprost (Lumigan ${ }^{\circledR}$; Allergan Inc., Irvine CA), latanoprost (Xalatan ${ }^{\circledR}$; Pfizer, New York, NY), and travoprost (Travatan ${ }^{\circledR}$; Alcon Laboratories, Fort Worth, TX) are commercially available agents in this class. Latanoprost was the first prostaglandin to be approved for use in glaucoma and is the most prescribed agent in its class. Most prostaglandin mimetics, including latanoprost, appear to effect a hypotensive response by remodeling the extracellular matrix of the ciliary body, thereby enhancing uveoscleral outflow. ${ }^{2,3}$ Bimatoprost is unique, in that it also has an effect on the conventional pathway. ${ }^{4}$ Thus, bimatoprost has a dual mechanism of action, whereby it lowers IOP by acting on both the pressure-sensitive and -insensitive outflow pathways. ${ }^{5}$

Short-term comparative studies of the IOP-lowering effect of latanoprost, travoprost, and bimatoprost have shown statistical similarity and, in some cases with bimatoprost, a superior effect. ${ }^{6-9}$ When IOP lowering with a prostaglandinclass agent is inadequate, the usual practice is to add an adjunctive IOP-lowering agent belonging to another class or to switch to one or more agents from the same class. ${ }^{10}$ While some short-term studies have shown improved IOP lowering when patients who had poor IOP-lowering response to latanoprost were switched to bimatoprost, ${ }^{11,12}$ long-term data are scarce.

We retrospectively evaluated long-term IOP lowering in poor responders to latanoprost in our practice who were

\footnotetext{
${ }^{1}$ Department of Ophthalmology, University of Illinois at Chicago, Chicago, IL.

${ }^{2}$ Midwest Eye Center SC, Calumet City, IL.

This paper was presented, in part, at the Annual Meeting of the American Academy of Ophthalmology, November 11-14, 2006, Las Vegas, NV.

The authors have no proprietary interest in any of the products mentioned in this paper. This physician-initiated study was not supported by any grant.

Sriram Sonty has served as a consultant, speaker, and investigator and received research and travel grants from Alcon Inc., Allergan Inc., Merck \& Co Inc., and Pfizer Inc.
} 
Table 1. Ophthalmic Side-Effects of Prostaglandin INTRAOCUlAR-Pressure LOWERING AgENTS ${ }^{a}$

\begin{tabular}{lc}
\hline Ophthalmic side-effects & Reversibility (yes/no) \\
\hline Eyelash: hypertrichosis & Yes \\
Periocular pigmentation & Yes \\
Conjunctival hyperemia & Yes \\
Ocular surface changes/allergy/blepharitis & Yes \\
Iris pigmentary changes (darkening) & No \\
Uveitis & Yes \\
Cystoid macular edema & Yes \\
\hline
\end{tabular}

aAdapted from Reference 13.

switched to, and maintained on, bimatoprost. Patients who had $<20 \%$ IOP lowering from untreated baseline after the initiation of ocular hypotensive therapy were considered to be "not at target IOP." This retrospective review was done as a pilot study preparatory to a long-term prospective study evaluating IOP lowering with bimatoprost in poor responders to latanoprost.

Our aim was to study long-term IOP lowering following a switch from latanoprost to bimatoprost in patients with OAG whose IOP was not at target while on latanoprost monotherapy or polytherapy with latanoprost and adjunctive agents.

\section{Methods}

We identified patients with OAG receiving latanoprost alone or as polytherapy with other IOP-lowering adjunctive agents who were switched to bimatoprost due to inadequate IOP lowering during the study period-December 1, 2002 to February 28, 2005-by a retrospective chart review.

All patients included in the study signed an "Authorization and Consent to Disclose Medical Information" form. In our practice, we routinely obtain written, informed consent from our patients to use information from their medical records for clinical research in accordance with the regulations of the Health Insurance Portability and Accountability Act (HIPAA). Thus, no separate investigational review board approval was deemed necessary for this pilot study.

\section{Inclusion criteria}

A decrease in IOP of $<20 \%$ from pretreatment baseline was considered to be "not at target." Patients with OAG whose IOP (as measured by Goldmann applanation tonometry) was "not at target" while on treatment with latanoprost either alone or with adjunctive agents and who were switched to bimatoprost during the study period were included.

\section{Exclusion criteria}

Since our objective was to assess long-term IOP lowering with bimatoprost, we included only patients who had follow-up records of at least 6 months following the switch. Eyes that had undergone filtering surgery or recent laser surgery ( 1 year prior to the initiation of bimatoprost therapy) were excluded from the analysis.

We documented IOP readings at 6, 12, and 24 months after the switch to bimatoprost. The main outcome assessed was mean IOP in both the right (OD) and left (OS) eyes at each time point studied and percent change in mean IOP from baseline before the switch to the end of the study.

\section{Safety}

We recorded discontinuations of bimatoprost and reasons for discontinuation as an assessment of safety. A note was made if any known side effects of topical therapy with bimatoprost were recorded in the chart (Table 1$).{ }^{13}$

\section{Statistical analyses}

For the efficacy analysis, OD and OS were analyzed separately to avoid between-eye correlations and data are presented as the mean IOP \pm standard deviation. The primary endpoint was to examine if bimatoprost contributed to further IOP lowering. Statistical significance was assessed by using paired Student $t$-tests. An alpha level of 0.05 was used as the criterion for significance. Statistical analysis was done by using Microsoft ${ }^{\circledR}$ Office Excel 2003 software (Microsoft Corp., Redmond, WA).

\section{Results}

We identified a total of 30 patients (30 eyes OD, 29 eyes OS) who fit the study criteria after a chart review and collected data from their medical records for analyses. Patient

Table 2. Baseline Characteristics of Study Patients

\begin{tabular}{|c|c|}
\hline \multicolumn{2}{|l|}{ Age (years) } \\
\hline Mean (range) & 70 years $(45-88)$ \\
\hline \multicolumn{2}{|l|}{ Sex, n $(\%)$} \\
\hline Male & $18(60)$ \\
\hline Female & $12(40)$ \\
\hline \multicolumn{2}{|l|}{ Race, n (\%) } \\
\hline African American & $20(67)$ \\
\hline White & $9(30)$ \\
\hline Hispanic & $1(3)$ \\
\hline \multicolumn{2}{|l|}{ IOP-lowering Rx, n (\%) } \\
\hline Latanoprost alone & $15(50)$ \\
\hline Latanoprost +1 Adjunctive $\mathrm{Rx}$ & $8(26.6)$ \\
\hline Latanoprost +2 Adjunctive $\mathrm{Rx}^{\mathrm{a}}$ & $4(13.3)$ \\
\hline Latanoprost +3 Adjunctive $\mathrm{Rx}^{\mathrm{a}}$ & $3(10)$ \\
\hline \multicolumn{2}{|l|}{ Baseline IOP } \\
\hline Right eye & $23.1 \pm 4.3 \mathrm{mmHg}$ \\
\hline Left eye & $22.3 \pm 3.8 \mathrm{mmHg}$ \\
\hline
\end{tabular}


Table 3. Details of Adjunctive Intraocular-Pressure-Lowering Therapy Used by Study Subjects

\begin{tabular}{lc}
\hline Adjunctive IOP-lowering Rx & No. of patients \\
\hline Brimonidine purite 0.15\% & 6 \\
Dorzolamide hydrochloride 2\%-timolol maleate 0.5\% fixed combination & 5 \\
Brinzolamide 1\% & 3 \\
Timolol gel (timolol maleate GS 0.5\%) & 2 \\
Timolol hemihydrate 0.5\% & 2 \\
Dorzolamide hydrochloride 2\% & 1 \\
Pilocarpine 2\% & 1 \\
\hline
\end{tabular}

demographics are detailed in Table 2. All patients except 1 were over 50 years old (mean, 70) and most of the patients (67\%) were African American. One (1) eye of 1 patient was excluded due to previous filtering surgery.

Half the study population $(n=15)$ was on monotherapy with latanoprost when they were switched to bimatoprost. The rest of the patients $(n=15)$ were on 1 or more topical adjunctive IOP-lowering agents along with latanoprost prior to the switch to bimatoprost. These patients continued the adjunctive treatments along with bimatoprost postswitch. Table 3 shows the details of adjunctive IOP-lowering therapies used by the patients. Brimonidine purite $0.15 \%$ (Alphagan- $\mathrm{P}^{\circledR}$; Allergan Inc.) and dorzolamide/timolol fixed combination (Cosopt ${ }^{\mathrm{TM}}$; Merck \& Co., Whitehouse Station, NJ) were the most common adjunctive agents used. Patients received latanoprost for at least 4 weeks before the switch. All patients were followed for a minimum period of 12 months.

\section{Efficacy}

Mean IOP prior to the switch to bimatoprost was $23.1 \pm$ $4.3 \mathrm{mmHg}$ in OD and $22.3 \pm 3.8 \mathrm{mmHg}$ in OS. IOP was lower in both OD and OS following the switch at all time points studied (Fig. 1). Mean IOP in OD was $19.2 \pm 3.0 \mathrm{mmHg}$ at 6 months, $18.0 \pm 3.8 \mathrm{mmHg}$ at 12 months, and $18.2 \pm 4.0$ $\mathrm{mmHg}$ at 24 months $(P<0.005$ at all time points studied). In OS, the mean IOP values for the above time points were $18.8 \pm 3.3 \mathrm{mmHg}$ (6 months), $18.4 \pm 3.4 \mathrm{mmHg}$ (12 months), and $17.0 \pm 3.2 \mathrm{mmHg}$ (24 months); the difference in mean IOP was significantly lower than at the preswitch baseline $(P<0.005)$ at all time points.

Following the switch to bimatoprost, the additional percent of IOP lowering ranged from 17.8 to $22.0 \%$ in OD and from 15.0 to $24.0 \%$ in OS. Of note, no further adjunctive IOPlowering therapy was added to the regimen of any patient after the switch to bimatoprost.

\section{Safety}

Only 1 patient stopped using bimatoprost due to an adverse event. This patient developed periocular hyperpigmentation after 6 months and opted to stop using bimatoprost in spite of good IOP control. Nine (9) patients were lost to follow-up after 12 months due to changes in their insurance coverage and transfer to other practices.

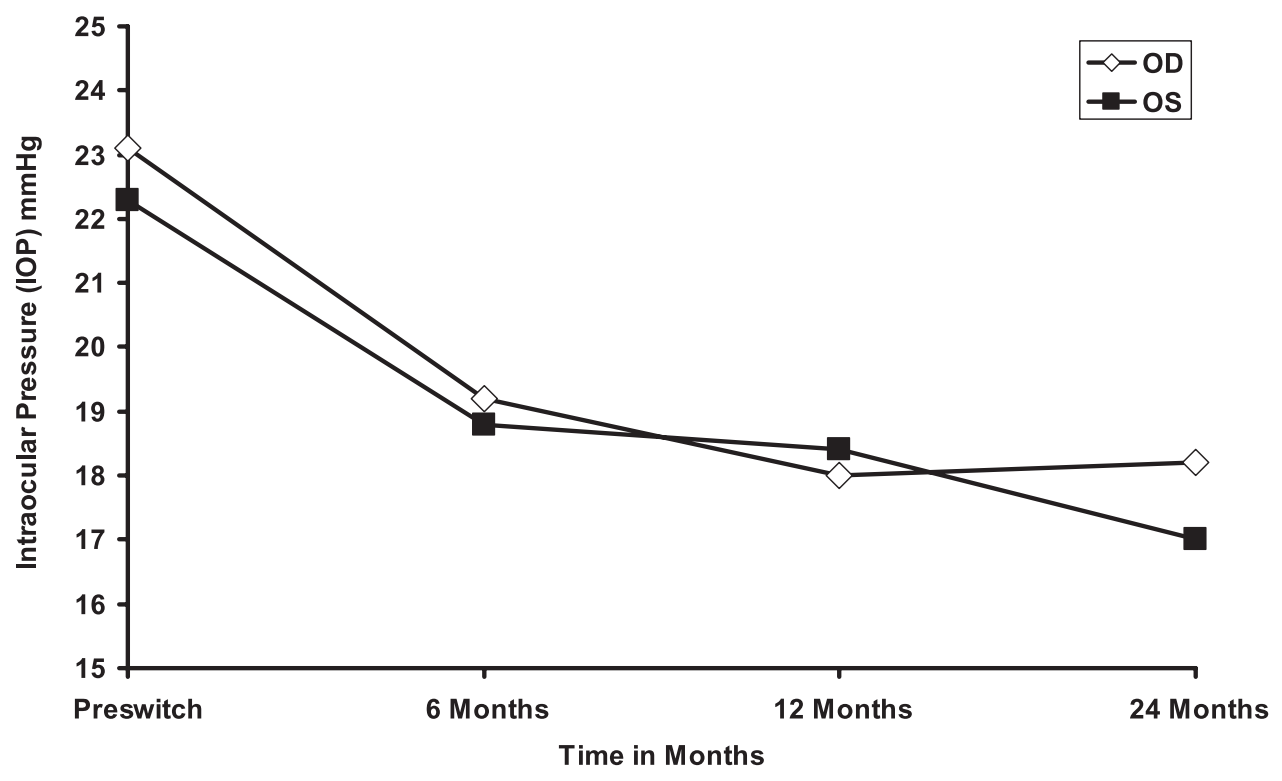

FIG. 1. Reduction in mean intraocular pressure (IOP) in the right and left eyes following a switch to bimatoprost. *Significant $(P<0.005)$ additional IOP reduction after switch to bimatoprost at all time points studied. 


\section{Discussion}

Of the 3 first-line agents in the prostaglandin class, latanoprost has been in use the longest and continues to be the most prescribed medication for IOP lowering in patients with glaucoma. Short-term studies ( $\leq 3$ months) have shown that bimatoprost can provide additional IOP lowering, when compared with latanoprost. ${ }^{11,12,14}$ In earlier studies, the additional IOP-lowering effect of bimatoprost was seen both in latanoprost responders as well as in patients who responded poorly to latanoprost, suggesting a superior IOP-lowering effect with bimatoprost, compared with latanoprost. It may be argued that improved compliance, regression to the mean, or a Hawthorne effect ${ }^{15}$ may have contributed to the shortterm additional IOP-lowering effect with bimatoprost seen in earlier studies comparing bimatoprost with latanoprost. But clinical evidence of sustained long-term (3 years) IOP lowering with bimatoprost was demonstrated in a prospective study when used as monotherapy in treatment naïve glaucoma patients. ${ }^{16}$ Our study suggests that significant sustained long-term IOP lowering also occurs with switching to bimatoprost in patients who are poor responders to latanoprost. The added hypotensive effect of bimatoprost observed in our study may be due to differences in mechanisms of action of the 2 agents. Evidence to support the hypothesis that bimatoprost interacts with a prostamide receptor in the trabecular meshwork to enhance outflow was recently published, where bimatoprost was shown to increase outflow facility by the conventional pathway by almost $50 \%$ in a human anterior segment perfusion model. ${ }^{4}$

In general, the prostaglandin-class agents have a similar adverse-effect profile, but the frequency of side effects varies from drug to drug. ${ }^{8,13}$ It has been reported that the ocular tolerability of bimatoprost is less, when compared with latanoprost. ${ }^{9}$ However, in our series, all but 1 patient tolerated bimatoprost well and continued on the drug during the 24month study period. Only 1 patient in our series discontinued bimatoprost due to a side effect.

\section{Conclusions}

While additional long-term prospective studies are called for to confirm the findings of our small retrospective study, our results support switching latanoprost-poor responders to bimatoprost before adding a new adjunctive agent from another class to latanoprost therapy. The benefits of this strategy include sustained IOP lowering with the continued simplicity of the regimen, ensuring better patient compliance, cost savings by avoidance of polytherapy, and delaying other interventions, such as laser treatments or surgery. (Word Count: 1544)

\section{Acknowledgments}

The authors thank Vasantha Reddi, PhD, for reviewing the statistical analysis and Mini Balaram, MD, for assistance with manuscript preparation.

\section{References}

1. Quigley, H.A., and Broman, A.T. The number of people with glaucoma worldwide in 2010 and 2020. Br. J. Ophthalmol. 90:262-267, 2006.

2. Lindsey, J.D., Kashiwagi, K., Kashiwagi, F., et al. Prostaglandins alter extracellular matrix adjacent to human ciliary muscle cells in vitro. Invest. Ophthalmol. Vis. Sci. 38:22142223, 1997.

3. Weinreb, R.N., and Lindsey, J.D. Metalloproteinase gene transcription in human ciliary muscle cells with latanoprost. Invest. Ophthalmol. Vis. Sci. 43:716-722, 2002.

4. Wan, Z., Woodward, D.F., Cornell, C.L., et al. Bimatoprost, prostamide activity, and conventional drainage. Invest. Ophthalmol. Vis. Sci. 48:4107-4115, 2007.

5. Brubaker, R.F. Mechanism of action of bimatoprost (Lumigan). Surv. Ophthalmol. 45(Suppl 4):S347-S351, 2001.

6. Walters, T.R., DuBiner, H.B., Carpenter, S.P., et al. 24-hour IOP control with once-daily bimatoprost, timolol gel-forming solution, or latanoprost: A 1-month, randomized, comparative clinical trial. Surv. Ophthalmol. 49(Suppl 1):S26-S35, 2004.

7. Noecker, R.S., Dirks, M.S., Choplin, N.T., et al. A six-month, randomized, clinical trial comparing the intraocular pressure-lowering efficacy of bimatoprost and latanoprost in patients with ocular hypertension or glaucoma. Am. J. Ophthalmol. 135:55-63, 2003.

8. Gandolfi, S., Simmons, S.T., Sturm, R., et al. Three-month comparison of bimatoprost and latanoprost in patients with glaucoma and ocular hypertension. Adv. Ther. 18:110-121, 2001.

9. Parrish, R.K., Palmberg, P., and Sheu, W.P. A comparison of latanoprost, bimatoprost, and travoprost in patients with elevated intraocular pressure: A 12-week, randomized, masked-evaluator multicenter study. Am. J. Ophthalmol. 135:688-703, 2003.

10. Martinez, A., and Sanchez, M. Intraocular pressure-lowering effect of dorzolamide/timolol fixed combination in patients with glaucoma who were unresponsive to prostaglandin analogs/prostamides. Curr. Med. Res. Opin. 23:595-599, 2007.

11. Williams, R.D. Efficacy of bimatoprost in glaucoma and ocular hypertension unresponsive to latanoprost. Adv. Ther. 19:275-281, 2002.

12. Gandolfi, S.A., and Cimino, L. Effect of bimatoprost on patients with primary open-angle glaucoma or ocular hypertension who are nonresponders to latanoprost. Ophthalmology 110:609-614, 2003.

13. Hollo, G. The side effects of the prostaglandin analogues. Exp. Opin. Drug Safety 6:45-52, 2007.

14. Law, S.K., Song, B.J., Fang, E., et al. Feasibility and efficacy of a mass switch from latanoprost to bimatoprost in glaucoma patients in a prepaid health maintenance organization. Ophthalmology 112:2123-2130, 2005.

15. McCarney, R., Warner, J., Iliffe, S., et al. The Hawthorne effect: A randomised, controlled trial. BMC Med. Res. Methodol. 7:30, 2007.

16. Gupta, V., Srinivasan, G., Sharma, A., et al. Comparative evaluation of bimatoprost monotherapy in primary chronic angle closure and primary open-angle glaucoma eyes: A three-year study. J. Ocul. Pharmacol. Ther. 23:351-358, 2007.

Received: March 11, 2008 Accepted: July 3, 2008

Reprint Requests: Sriram Sonty Midwest Eye Center SC 1700 East West Road Calumet City, IL 60409

E-mail: ssonty@msn.com 